\title{
A Case of Pulmonary Embolism on Dabigatran
}

\author{
Pragati Tandon ${ }^{\mathrm{a}}$, Sameer Khanijo ${ }^{\mathrm{a}, \mathrm{b}}$
}

\begin{abstract}
It is well known that hospitalized medical patients are at higher risk for the development of venous thromboembolism. As a result patients are placed on standard prophylactic anticoagulation. As newer anticoagulants are coming to the market patients on these novel oral agents are continued on them instead of being transitioned to the standard therapies.Dabigatran, one of the newer agents, is an oral direct thrombin inhibitor (DTI) approved for anticoagulation in non-valvular atrial fibrillation and DVT prophylaxis in post-operative patients after knee and hip surgeries. Dabigatran is an effective anticoagulant because DTI's suppress thrombus growth [1]. We report a case of extensive pulmonary emboli in a patient taking dabigatran. Although dabigatran is not currently approved for DVT/VTE prophylaxis in the hospitalized medical patient, recent studies demonstrate its non-inferiority compared with warfarin in the prevention of recurrent VTE. In light of this, we argue that our patient constitutes a treatment failure.
\end{abstract}

Keywords: Pulmonary embolism; Oral anticoagulants; Thromboprophylaxis; Dabigatran

\section{Introduction}

We report a case of extensive pulmonary emboli in a patient taking dabigatran. Although dabigatran is not currently approved for DVT/VTE prophylaxis in the hospitalized medical patient, recent studies demonstrate its non-inferiority compared with warfarin in the prevention of recurrent VTE. In light of this, we argue that our patient constitutes a treat-

\footnotetext{
Manuscript accepted for publication July 5, 2013

${ }^{a}$ Hofstra North Shore LIJ School of Medicine Residency Program in Internal Medicine, Hempstead, NY, USA

${ }^{\mathrm{b}}$ Corresponding author: Sameer Khanijo, Department of Internal Medicine, 300 Community Drive, 4DSU, Office of Graduate Medical Education, Manhasset, NY 11030, USA. Email: skhanijo2@nshs.edu
}

doi: http://dx.doi.org/10.4021/jmc1400w ment failure.

\section{Case Report}

A 65 year-old man with a history of CAD s/p CABG, HTN, HLD, biV-ICD and atrial fibrillation on dabigatran was initially admitted for a right inguinal hernia surgery. His course was complicated by sepsis, respiratory failure requiring intubation and ventricular tachycardia which required antiarrhythmics. He was ultimately stabilized and extubated. He improved and was ambulating for several days. On the scheduled day of discharge, he was noted to be hypoxic to $86 \%$ on room air. He had mild crackles but no cough, lower extremity edema or JVD. As the patient had a complicated and lengthy hospital course, with sudden onset hypoxia, a CTPA was done to evaluate for pulmonary embolism which showed extensive pulmonary emboli bilaterally, without evidence of right heart strain.

\section{Discussion}

Dabigatran is an oral direct thrombin inhibitor (DTI) approved for anticoagulation in non-valvular atrial fibrillation and DVT prophylaxis in post-operative patients after knee and hip surgeries. Dabigatran is an effective anticoagulant because DTI's suppress thrombus growth by inhibiting both fibrin-bound and free thrombin which converts fibrinogen to fibrin [1]. Direct thrombin inhibitors are gaining favor because they require less frequent monitoring, have fewer interactions with foods and have a less variable drug availability when given twice daily [2].

In 2009, the RE-LY trial compared dabigatran and warfarin in patients with atrial fibrillation for stroke prevention. The primary outcome was acute stroke or systemic embolism. RE-LY studied dabigatran at doses of $110 \mathrm{mg}$ and 150 $\mathrm{mg}$ twice daily compared with warfarin dosed to an appropriate INR. The study found that low dose dabigaran was non-inferior to warfarin and high dose dabigatran reduced risk of stroke without increasing the risk of major bleeding. This formed the basis of FDA approval for the use of dabiga- 
tran in patients with non-valvular atrial fibrillation [2].

Later that year, RE-COVER evaluated dabigatran versus warfarin in the treatment of acute venous thromboembolism. This trial compared twice daily dabigatran and warfarin dosed to an appropriate INR with primary outcome of recurrent symptomatic, objectively confirmed venous thromboembolism and related death. Non-inferiority was demonstrated with a similar bleeding risk and safety profile. This study concluded that for the prevention of recurrent thromboembolism, dabigatran is as effective as warfarin and is associated with less bleeding [3].

Other DTIs such as ximelagatran have also been shown to be as effective as the standard regimen for the initial treatment of venous thromboembolism [4]. However, ximelagatran proved to be hepatotoxic. Although the FDA has not currently approved dabigatran for the treatment of acute venous thromboembolism, the effectiveness of other DTIs coupled with the promising results of the RE-COVER trial support that dabigatran can potentially serve as a therapeutic anticoagulant for this indication.

Under this premise, our patient would be considered a treatment failure of dabigatran. Despite compliance with an appropriate dose of dabigatran, our patient developed symptomatic bilateral pulmonary emboli. As oral direct thrombin inhibitors such as dabigatran gain broader application, clinicians should remain aware that treatment failures are possible and when symptomatology indicates, appropriate investigation to rule out clotting should be undertaken to prevent significant patient morbidity and mortality.

\section{References}

1. Gross PL, Weitz JI. New antithrombotic drugs. Clin Pharmacol Ther. 2009;86(2):139-146.

2. Connolly SJ, Ezekowitz MD, Yusuf S, Eikelboom J, Oldgren J, Parekh A, Pogue J, et al. Dabigatran versus warfarin in patients with atrial fibrillation. $\mathrm{N}$ Engl J Med. 2009;361(12):1139-1151.

3. Schulman S, Kearon C, Kakkar AK, Mismetti P, Schellong S, Eriksson H, Baanstra D, et al. Dabigatran versus warfarin in the treatment of acute venous thromboembolism. N Engl J Med. 2009;361(24):2342-2352.

4. Fiessinger JN, Huisman MV, Davidson BL, Bounameaux H, Francis CW, Eriksson H, Lundstrom T, et al. Ximelagatran vs low-molecular-weight heparin and warfarin for the treatment of deep vein thrombosis: a randomized trial. JAMA. 2005;293(6):681-689. 